\title{
A NOTE ON THE SPAN OF TRANSLATIONS IN $L^{p}$
}

\author{
C. S. HERZ 1
}

Suppose $f \in L^{1} \cap L^{p}$. $f$ is said to have the Wiener closure property, ${ }^{2}$ $(C)$, if the translates of $f$ span $L^{p}$. Since $f \in L^{1}$, the Fourier transform $\hat{f}$ is well defined. Let $Z(f)$ be the set of zeros of $\hat{f}$. One would like to reformulate $(C)$ in terms of structural properties of the closed set $Z(f)$. The problem seems quite difficult; in this note we show that $(C)$ is nearly equivalent to a uniqueness property of $Z(f){ }^{3}$

It is assumed that the notion of the spectrum ${ }^{4}$ of a bounded continuous function is familiar.

Definition. A closed set is of type $U^{a}$ if the only bounded continuous function in $L^{q}$ with spectrum contained in the set is the null function. ${ }^{5}$

We shall say that $f$ has property $(U)$ if $Z(f)$ is of type $U^{q}$ where $1 / p+1 / q=1$. Pollard, [4], has observed, what is true for any locally compact Abelian group, that

Theorem 1. For $1 \leqq p<\infty,(U)$ implies $(C)$.

In the converse direction one has trivially,

TheOREM 2. For $2 \leqq p<\infty,(C)$ implies $(U)$.

Of course, this also holds for $p=1$. What is left open is the case $1<p<2$. Here we have two classes of results corresponding to weakening the conclusion and strengthening the hypothesis respectively.

Definition. A closed set is of type $U^{q^{*}}$ if there is no nontrivial complex measure of bounded variation with spectrum (support) in the set whose Fourier-Stieltjes transform belongs to $L^{q}$.

Presented to the Society, October 27, 1956 under the title The closure of translations in $L^{p}$; received by the editors June 27, 1956 and, in revised form, October 3, 1956.

1 The research for this paper was supported by the United States Air Force under Contract No. AF18(600)-685 monitored by the Office of Scientific Research.

${ }^{2}$ For a general discussion of the problem see [6] of the bibliography. The reference is to Part III, $\$ 2$.

3 This is the viewpoint of [4].

4 For an elaborate treatment of spectral theory see [3]; however [5] will be more accessible to the classical analyst. For assertions about the spectrum not proved in the text see these references.

5 The definition here is equivalent to that in [4]; the proof of equivalence is essentially the same as the proofs given there. Simple modifications of the method show that sets of uniqueness can be defined using any of a large variety of summability methods, including, when $q<\infty$, ordinary convergence of trigonometric integrals. 
We shall say that $f$ has property $\left(U^{*}\right)$ if $Z(f)$ is of type $U^{q^{*}}$ where $1 / p+1 / q=1$.

\section{Theorem $2^{*}$. For $1 \leqq p<\infty,(C)$ implies $\left(U^{*}\right)$.}

The only result which requires any imagination is the next. It should be noted that the proof makes only trivial use of the "natural" assumption, $f \in L^{p}$, but it depends strongly on the fact that $f \in L^{1}$.

Theorem 3. If for some $\epsilon>0, \hat{f} \in \operatorname{Lip} \epsilon$, then $(C)$ implies $(U)$.

We remark that the extra hypothesis is certainly fulfilled ${ }^{6}$ if $\int|f(x)||x|{ }^{\star} d x<\infty$.

To prove the above theorems, first observe that $(C)$ is equivalent to the statement: if $\phi \in L^{q}$ and the convolution $f * \phi=0$, then $\phi=0$. Let $g \in L^{1}$ be such that $\hat{g}$ vanishes outside a compact set. If $f * \phi=0$ then $f *(g * \phi)=0$ while on the other hand, $\phi=0$ if and only if $g * \phi=0$ for each such $g$. Thus we may replace $\phi$ if necessary by $g * \phi$ and consider only bounded continuous functions $\phi \in L^{q}$ with compact spectrum $\Lambda(\phi)$. The defining property of the spectrum is that $f * \phi=0$ implies $\Lambda(\phi) \subset Z(f)$; this proves Theorem 1 . The propositions in the converse direction are argued by contradiction. We assume there exists some non-null $\phi \in L^{q}$ with $\Lambda(\phi) \subset Z(f)$ and wish to prove that $f * \phi=0$, or something just as good. This is essentially a spectral synthesis problem, and as such it appears to require extra conditions. For example if $\phi$ is known to be a Fourier-Stieltjes transform, $\Lambda(\phi) \subset Z(f)$ implies $f * \phi=0$; this establishes Theorem $2 *$. The observation that it suffices to consider $\phi$ 's with compact spectrum shows that for $1 \leqq q \leqq 2$, type $U^{q}$ is identical with type $U^{q^{*}}$ since every $\phi \in L^{q}$ with compact spectrum is a Fourier transform. Theorem 2 is therefore an immediate corollary of Theorem $2 *$.

All the foregoing is valid for locally compact Abelian groups. However, for simplicity, we present the details of the proof of Theorem 3 only for the real line. The extension to the general case is clearly indicated in [3], (cf. the proof there of Lemma 4.4). Suppose $Z(f)$ is not of type $U^{q}$. Then there is a non-null $\phi \in L^{q}$ with compact spectrum $\Lambda(\phi) \subset Z(f)$. Let $f^{(n)}$ denote the convolution of $f$ with itself $n$ times. If we can show that $f^{(n)} * \phi=0$ for some $n$ we are through, for let $n$ be the first integer for which this is true. If $n=1$, fine! Otherwise $f^{(n-1)} * \phi$ is a non-null function in $L^{q}$ with spectrum $\Lambda\left(f^{(n-1)} * \phi\right)$ $\subset \Lambda(\phi) \subset Z(f)$ and $f *\left(f^{(n-1)} * \phi\right)=0$. The Lipschitz condition is just what we need to guarantee the existence of an $n$ so that $f^{(n)} * \phi=0$.

Choose an $h>0$ and set $k(x)=(x / 2)^{-2} \sin ^{2} x / 2$. Define $\Phi_{h}(t)$

- Theorem 3 is supposed to compare favorably with Theorem B of [4]. 
$=(2 \pi)^{-1} \int \exp (-i t x) k(h x) \phi(x) d x$. Then $\Phi_{h}$ vanishes outside the set $\Lambda^{h}$ consisting of those points at a distance $<h$ from $\Lambda(\phi)$. Moreover $f^{(n)} * \phi(x)=\int f^{(n)}(x-y) \phi(y) d y=\lim _{h \rightarrow 0} \int f^{(n)}(x-y) k(h y) \phi(y) d y$ $=\lim _{h \rightarrow 0} \int \exp (i t x) \hat{f}^{n}(t) \Phi_{h}(t) d t$. Hence it suffices to prove that $\int\left|\hat{f}^{n}(t) \Phi_{h}(t)\right| d t=o(1)$ as $h \rightarrow 0$. Now $\hat{f}$ vanishes on $\Lambda(\phi)$ and $\hat{f} \in \operatorname{Lip} \epsilon$. Hence if $t$ is within $h$ of $\Lambda(\phi)$, i.e., $t \in \Lambda^{h}, \hat{f}(t)=O\left(h^{\mathrm{c}}\right)$. Since the integration is extended only over $\Lambda^{h}, \int\left|\hat{f}^{n}(t) \Phi_{h}(t)\right| d t=O\left(h^{n e}\right) \int\left|\Phi_{h}(t)\right| d t$. The last integral obviously is $O\left(h^{-\delta}\right)$ for some $\delta$ (a careful estimate will be considered later) so choose $n>\delta / \epsilon$.

The question of the structure of sets of type $U^{q}$ is quite open. Let $T$ be a closed set and $|T|$ its Lebesgue measure. Obviously $|T|=0$ is, in case $q \leqq 2$ a sufficient, and in case $q \geqq 2$ a necessary condition that $T$ be of type $U^{q}$. Exact criteria are available for $q=1$ ( $T$ has empty interior), $q=2(|T|=0)$, and $q=\infty$ ( $T$ is empty). One would like to interpolate. The next theorem is a step in that direction which gives some content to Theorem 1 . We consider $r$-tuple trigonometric series or integrals. $\Lambda^{h}$ has the same meaning as in the paragraph above, and $\operatorname{dim} T$ is the Hausdorff dimension of $T$.

TheOREM 4. Alternative sufficient conditions that the closed set $T$ be of type $U^{q}, q \geqq 2$ are

(i) $\left|\Lambda^{h}\right|=o\left(h^{r(1-2 / q)}\right)$ for each compact subset $\Lambda$ of $T$,

(ii) $\operatorname{dim} T<2 r / q$, with the proviso, if $r>2$, that $q \leqq 2 r /(r-2)$.

We shall give the proof of (i) only for ordinary trigonometric integrals. It suffices to show that if $\phi \in L^{q}$ is a bounded continuous function with compact spectrum $\Lambda(\phi) \subset T$ then $\phi=0$. This will be true if, in the previous notation, $\int\left|\Phi_{h}(t)\right| d t=o(1)$ as $h \rightarrow 0$. Using the Schwarz inequality, $\left\{\int\left|\Phi_{h}(t)\right| d t\right\}^{2} \leqq\left|\Lambda^{h}\right| \cdot \int\left|\Phi_{h}(t)\right|^{2} d t$. Next we employ the Parseval relation and the Hölder inequality.

$$
\begin{aligned}
\int\left|\Phi_{h}(t)\right|^{2} d t & =\int|k(h x) \phi(x)|^{2} d x \\
& \leqq\left\{\int \mid k(h x)^{2 q /(q-2)} d x\right\}^{1-2 / q} \cdot\left\{\int|\phi(x)|^{q} d x\right\}^{2 / q} \\
& =O\left(h^{-1+2 / q}\right) \cdot O(1) .
\end{aligned}
$$

Combining the estimates, $\left\{\int\left|\Phi_{h}(t)\right| d t\right\}^{2}=\left|\Lambda^{h}\right| \cdot O\left(h^{-1+2 / q}\right)=o(1)$ since $\left|\Lambda^{h}\right|=o\left(h^{1-2 / q}\right)$ by hypothesis. (ii) was proved by Beurling [1] for $r=1$ and extended by Deny [2, pp. 144-145].

The conditions of Theorem 4 are clearly unnecessary since an ordinary set of uniqueness is of type $U^{q}$ for every $q, 1 \leqq q<\infty$. However the estimates cannot be improved. 
In conclusion we mention one amusing problem for $r$-dimensional Euclidean space. Suppose $f \in L^{p}$ and vanishes outside a compact set. Then $\hat{f}$ is an entire function of exponential type. For $r=1$, the translates of $f$ span $L^{p}$ for all $p, 1<p<\infty$, since $Z(f)$ is countable. However consideration of a few Bessel functions leads to the conclusion that for $r>1$ the theorem is certainly false unless $p \geqq 2 r /(r+1)$. Is this a sufficient condition? Posing the problem otherwise, for what $q$ is the set of real zeros of an entire function of exponential type in $r$-variables necessarily of type $U^{a}$ ?

\section{BIBLIOGRAPHY}

1. Arne Beurling, On a closure problem, Arkiv för Matematik vol. 1 (1951) pp. 301-303.

2. Jacques Deny, Les potentials d'energie finie, Acta Math. vol. 83 (1950) pp. 107183.

3. C. S. Herz, The spectral theory of bounded functions, to appear.

4. Harry Pollard, The closure of translations in $L^{p}$, Proc. Amer. Math. Soc. vol. 2 (1951) pp. 100-104.

5. - The harmonic analysis of bounded functions, Duke Math. J. vol. 20 (1953) pp. 499-512.

6. I. E. Segal, The group algebra of a locally compact group, Trans. Amer. Math. Soc. vol. 61 (1947) pp. 69-105.

Cornell University 